\title{
Some Comments on Multigrid Methods for Computing Propagators
}

\author{
Alan D. Sokal \\ Department of Physics \\ New York University \\ 4 Washington Place \\ New York, NY 10003 USA \\ SOKAL@NYU.EDU
}

July 27, 1993

\begin{abstract}
I make three conceptual points regarding multigrid methods for computing propagators in lattice gauge theory: 1) The class of operators handled by the algorithm must be stable under coarsening. 2) Problems related by symmetry should have solution methods related by symmetry. 3) It is crucial to distinguish the vector space $V$ from its dual space $V^{*}$. All the existing algorithms violate one or more of these principles.
\end{abstract}


There has recently been much interest in developing multigrid methods for solving large linear systems with disordered coefficients [1, 2, 3], and in particular for computing the bosonic or fermionic propagator in a background gauge field. Many interesting ideas in this direction have been proposed by the Amsterdam [4, 5, 6, 0, 8, 9, 10, Boston [11, 12, 13, 14, 15], Hamburg [16, 17, 18, 19, 20, 21, 22, 23, 24, 25] and Israeli [26, 27, 28, 29, 30, 31, 32, 33, 34, 35] groups. However, all these discussions have missed what I consider to be some key conceptual points. Perhaps a brief note explaining these points is therefore warranted.

First point. We are interested in solving linear systems of the form $A x=b$ for some class of linear operators $A$. So the first order of business must be to specify clearly the class $\mathcal{C}$ of operators to which our algorithm is intended to apply. This is not a completely trivial matter, because

1) For a multigrid (as opposed to two-grid) algorithm to be well-defined, the class $\mathcal{C}$ must be stable under coarsening. [

In other words, we start with some class $\mathcal{C}_{0}$ of operators on the finest grid, for which we want to solve $A x=b$. But then our multigrid algorithm produces operators on the first coarse grid, which belong to some quite possibly larger class $\mathcal{C}_{1}$ - what this class is depends on our procedure for choosing interpolation, restriction and coarse-grid operators. If we are then to continue to the second coarse grid, this procedure must be defined for all the operators in this larger class. If we want to allow an arbitrary number of grids, then we cannot stop short of a class $\mathcal{C} \supset \mathcal{C}_{0}$ that is stable under coarsening.

For example, our initial interest might be in nearest-neighbor Laplace operators in an $S U(N)$ gauge field, with $x$-independent values for the hopping parameter $\beta$ and the mass $m$ :

$$
\mathcal{C}_{0}=\left\{A \equiv-\beta \Delta_{U}+m^{2}: U_{x y} \in S U(N), \beta \geq 0, m^{2} \in \mathbb{R}, A>0\right\}
$$

where

$$
\left(\Delta_{U}\right)_{x y}= \begin{cases}-2 d & \text { for } x=y \\ U_{x y} & \text { for }|x-y|=1 \\ 0 & \text { otherwise }\end{cases}
$$

But quite possibly our coarsening procedure will produce a larger class of operators on the coarse grids: the mass may vary from site to site, or may even become nontrivial in color space; and the off-diagonal elements may have magnitudes that vary

\footnotetext{
1 Typically the gauge field $U$ is chosen stochastically from some probability distribution $P(U)$, e.g. either that of quenched lattice gauge theory or else that of lattice gauge theory with dynamical fermions.

${ }^{2}$ I emphasize that the issue here is not whether the algorithm performs well or poorly; the issue is whether the algorithm is meaningful at all.
} 
from link to link, or they may even leave the space $\mathbb{R}_{+} \times S U(N)$ and enter its linear

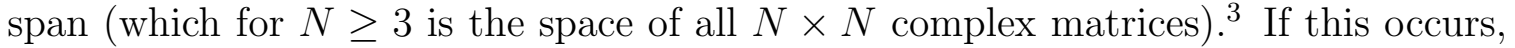
we must then go back to the beginning, and define our coarsening procedure for all the operators in this larger class - and so on successively until the class stabilizes.f

This comment is relevant to the ground-state-projection (GSP) multigrid algorithms proposed by the Boston [11, 12, 13, 14, 15] and Hamburg [17, 18, 19, 20, 21, 24, 25] groups, in which the range of the interpolation operator is spanned by the lowest eigenvectors of the operator $A$ modified to impose Neumann boundary conditions on $2 \times 2$ or $3 \times 3$ blocks. The trouble here is that the concept of "Neumann b.c.", though well-defined for operators of the class (11), has no unambiguous meaning (as far as I can tell) when the off-diagonal elements of $A$ are more general than (positive real number) $\times$ (unitary matrix). Possibly an appropriate definition can be found, but this is a highly nontrivial problem, particularly when the required covariances are taken into account (see the "second point" below). The situation is different for the Amsterdam version of GSP multigrid [4, 5, 6, 8, 9 (see also [17]), which employs Dirichlet b.c. on $2 \times 2$ blocks. This operator is well-defined in general: one simply sets to zero those off-diagonal elements of $A$ that cross block boundaries. On the other hand, it is unclear whether Dirichlet b.c. give a good interpolation: for example, in the case of the pure Laplacian $(U \equiv I)$, Dirichlet b.c. give a reasonable interpolation only on $2 \times 2$ blocks, where they are equivalent to Neumann b.c. plus a multiple of the identity matrix (and yield piecewise-constant interpolation); on larger blocks, Dirichlet b.c. yield sine-wave interpolation kernels, which do not perform well (because smooth functions, e.g. constants, cannot be interpolated accurately). This is not necessarily an argument against Dirichlet b.c. on $2 \times 2$ blocks, but it does raise doubts about whether the concept behind GSP using Dirichlet b.c. is correct.

Second point. Suppose that there is a transformation $T: \mathcal{C} \rightarrow \mathcal{C}$ that is "trivially calculable", in the sense that from the solution of $A x=b$ we can trivially determine (in a negligible CPU time) the solution of $T(A) x=b^{\prime}$, and conversely. Then any sensible method (whether multigrid or anything else) for solving problems in $\mathcal{C}$ ought to be covariant under $T$, in the sense that using the method to solve $A x=b$ ought to give exactly the same approximate solutions, in exactly the same CPU time, as transforming to $T(A) x=b^{\prime}$, using the method to solve this latter equation, and then transforming back. Why? Well, suppose it weren't so. Then we would have two alternative methods for solving $A x=b$ and $T(A) x=b^{\prime}$, and presumably one of them would be better than the other; we should then use this method for both equations,

${ }^{3}$ This is a "dielectric gauge field" in the language of Mack [36].

${ }^{4}$ This criterion is irrelevant in unigrid [37] approaches (as proposed e.g. in [22, 23]), since here the concept of a coarse-grid operator never arises: everything is done on the original (fine) grid. However, these approaches have a severe drawback: the interpolation kernel must be computed on arbitrarily big blocks (e.g. $2^{k}$ or $3^{k}$ for arbitrarily large $k$ ), and this can be prohibitive if the CPU time for this computation grows faster than linearly in the block volume. 
and discard the other method. The principle here is quite general, and has nothing to do per se with linear equations:

2) Problems related by symmetry should have solution methods related by symmetry. (A special case is: Symmetric problems should have symmetric solution methods.)

Of course, this principle is not always valid: there could arise, in principle, cases of "spontaneous symmetry breaking in algorithm space", in which each of a family of non- $T$-covariant algorithms would be better than the best $T$-covariant algorithm. But I find this possibility extremely unlikely in the present context.

This principle has powerful consequences in combination with the first point. For example, the class $\mathcal{C}_{0}$ defined above is mapped into itself under $S U(N)$ gauge transformations and under space-independent rescalings $A \rightarrow \lambda A$; and many workers have recognized that a good algorithm ought to be covariant under these transformations. But the larger class of operators produced by coarsening may well be mapped into itself by space-dependent rescalings $A_{x y} \rightarrow \lambda_{x} A_{x y} \lambda_{y}^{*}$ or even by $G L(N, \mathbb{C})$ gauge transformations $A_{x y} \rightarrow U_{x} A_{x y} U_{y}^{*}$ with $U_{x} \in G L(N, \mathbb{C})$. The multigrid algorithm ought then to be covariant under this larger group; but none of the published algorithms, to my knowledge, have this property.

Third point. My third point is more abstract, but it is related to the second one. Consider again $A x=b$. On both physical and mathematical grounds,

3) It is crucial to distinguish between the vector space $V$ of solutions $x$ and the vector space $W$ of "forcing terms" $b$. Đ

Thus, $x$ may be a displacement while $b$ is a force; $x$ may be a magnetization while $b$ is a magnetic field; and so forth. Most commonly in physical applications, we have $W=V^{*}=$ dual space of $V$ : that is, there is a natural nondegenerate bilinear mapping $V \times W \rightarrow \mathbb{R}$, which typically can be interpreted as an energy (force - displacement, magnetic field · magnetization, etc.). In this case the principle becomes

${ }^{5}$ The parallel-transported multigrid (PTMG) algorithm proposed by the Israeli group [26, 27, 28, 29, 30, 31, 32, 33, 34, 35 avoids this problem by forcing the coarse-grid operator to lie in the original class $\mathcal{C}_{0}$. The price paid is that the coarse-grid operator does not satisfy the Galerkin condition $A_{1}=R A_{0} P$ (see footnote 12 below for discussion of why the Galerkin condition is desirable). The divergent iteration observed at small fermion mass [27, 28, 29, 30, 34, 35] is probably related to this choice of a non-Galerkin coarse-grid operator.

${ }^{6}$ Two vector spaces of the same finite dimension are of course isomorphic, but they are not naturally isomorphic. Otherwise put, there are many linear bijections from $V$ to $W$, and if we want to single out one of them we must find a physical reason for doing so.

${ }^{7}$ I use the notations $\ell(x) \equiv\langle\ell, x\rangle \equiv\langle x, \ell\rangle$ to denote the natural bilinear mapping acting on $\ell \in V^{*}$ and $x \in V$. 
$\left.3^{\prime}\right)$ It is crucial to distinguish between the vector space $V$ and its dual space $V^{*}$. (In a language perhaps more familiar to physicists: it is crucial to distinguish between contravariant and covariant tensors.)

This principle is well known to differential geometers and general relativists, who have learned (since roughly the 1950's and 1960's, respectively) the advantages of a "coordinate-free" (basis-independent) notation. However, this principle is often forgotten by elementary-particle physicists, because we typically learned our linear algebra in the context of either quantum mechanics or matrix theory - each of which works not on a bare vector space $V$, but rather on a vector space $V$ equipped with additional structure:

(a) Quantum mechanics employs a Hilbert space, i.e. a vector space equipped with an inner product (positive-definite bilinear form on $V$ ). This inner product induces an identification map between $V$ and $V^{*}$. In quantum mechanics the inner product has, of course, a good physical interpretation (transition amplitude). In other contexts we should introduce such an identification operator only if it has likewise a good physical or mathematical raison d'être.

(b) Matrix theory is linear algebra referred to a particular basis - and this can be very misleading, unless the basis has been selected by some good physical or mathematical criterion. For example, if we have chosen particular bases for $V$ and $V^{*}$, then the matrix with entries $\delta_{i j}$ defines a particular identification map from $V$ to $V^{*}$; but this identification is not natural, i.e. it is of no more physical or mathematical interest than any other linear bijection from $V$ to $V^{*}$. (In different bases this same identification would have different matrix representations, not equal to $\delta_{i j}$.) In particular, it is misleading to employ the term "identity matrix" in this context; this term ought to be reserved for the matrix representation of the identity operator, which maps a space $V$ into itself. (This operator has the matrix representation $\delta_{i j}$ with respect to any basis.)

As soon as we distinguish between $V$ and $V^{*}$, we must distinguish two very different types of linear operators:

- Linear operators $A: V \rightarrow V$.

- Linear operators $Q: V \rightarrow V^{*}$ [or what is equivalent, bilinear forms $\widehat{Q}: V \times V \rightarrow$ $\mathbb{R}$. 9 .

\footnotetext{
8 The correspondence is $\widehat{Q}(x, y)=(Q x)(y)$ for $x, y \in V$.

${ }^{9}$ In the complex case one has to consider sesquilinear forms, and the role of $V^{*}$ is played by the antidual space (i.e. the space of all antilinear maps from $V$ to $\mathbb{C}$ ). For simplicity we shall henceforth restrict attention to the real case. This is no loss of generality, because a complex vector space of dimension $n$ can be considered as a real space of dimension $2 n$; and under this correspondence eigenvalues are preserved (more precisely, $a+b i$ becomes the pair $a \pm b i$ ), hermiticity corresponds to symmetry, etc.
} 
Only for operators from $V$ to itself can we discuss eigenvalues, spectral radius, trace, determinant, etc.; only for operators from $V$ to $V^{*}$ can we discuss symmetry (or hermiticity) and positive-(semi)definiteness. एण.'⿴囗十

Thus, in most physical contexts the Laplacian arises naturally as an operator from $V$ to $V^{*}$ : the corresponding quadratic form is the energy functional. It is thus meaningless to discuss the "eigenvalues" of such an operator. (What is actually meant by such discussions will be explained below.)

Let us examine the multigrid method in this context. We are given a fine-grid operator $A_{0}: V_{0} \rightarrow V_{0}^{*}$ - usually symmetric and positive-definite - for which we want to solve $A_{0} x=b$. We then introduce a coarse-grid space $V_{1}$, along with restriction, interpolation (prolongation) and coarse-grid operators. On what spaces do these operators act? Examination of the multigrid algorithm indicates that restriction is always applied to a residual (right-hand side); thus, $R: V_{0}^{*} \rightarrow V_{1}^{*}$. Similarly, interpolation is always applied to an error (left-hand side); thus, $P: V_{1} \rightarrow V_{0}$. Finally, the coarse-grid operator acts similarly to the fine-grid operator, i.e. $A_{1}: V_{1} \rightarrow V_{1}^{*}$. We thus have the following diagram:

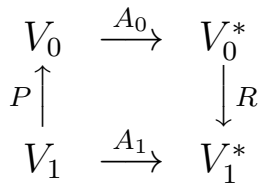

It follows that the oft-imposed condition $R=P^{*}$ is meaningful. Likewise, the

10 Symmetry means that $(Q x)(y)=(Q y)(x)$, or equivalently that $\widehat{Q}(x, y)=\widehat{Q}(y, x)$. Symmetry can also be written as $Q=Q^{*}$, once we remember the definition of dual operator: if $A: V \rightarrow W$, then $A^{*}: W^{*} \rightarrow V^{*}$ is defined by $\left(A^{*} x\right)(y)=x(A y)$ for $x \in W^{*}, y \in V$.

11 Positive-semidefiniteness means that $(Q x)(x) \geq 0$ for all $x \in V$. Positive-definiteness means that $(Q x)(x)>0$ for all $x \in V, x \neq 0$. 
Galerkin condition $A_{1}=R A_{0} P$ is meaningful. dition $R P=I$ [16, 17, 19, 22, 23] is meaningless.

However, this is not the end of the story, because the multigrid algorithm contains an additional ingredient, namely a relaxation (smoothing) operator $\mathcal{S}_{0}: V_{0} \times V_{0}^{*} \rightarrow V_{0}$ which takes an approximate solution of $A_{0} x=b$ and produces a new (and hopefully better) approximate solution. Usually this relaxation is a first-order stationary linear process

$$
\mathcal{S}_{0}(x, b)=S_{0} x+T_{0} b,
$$

where $S_{0}: V_{0} \rightarrow V_{0}, T_{0}: V_{0}^{*} \rightarrow V_{0}$ and the consistency condition is $T_{0}=\left(I-S_{0}\right) A_{0}^{-1}$ [verify that this formula is meaningful!]. Such a process ordinarily arises from a splitting

$$
A_{0}=B_{0}+C_{0}
$$

where $B_{0}: V_{0} \rightarrow V_{0}^{*}$ is some operator that is "easy to invert"; writing $A_{0} x=b$ as

${ }^{12}$ Briggs [38, pp. 67-69] and the Hamburg group [19, 22, 23] have given a very strong argument for the Galerkin choice of the coarse-grid operator. After fine-grid relaxation, we have an error $e_{0} \equiv x_{0}-A_{0}^{-1} b$ which satisfies the equation $A_{0} e_{0}=r_{0}$, where $r_{0} \equiv A_{0} x_{0}-b$ is the residual. Now, the multigrid philosophy is based on the idea that this error is smooth, in the sense that it is near to the range of the interpolation operator, i.e. $e_{0} \approx P e_{1}$ for some vector $e_{1} \in V_{1}$. This vector $e_{1}$ satisfies the equation $R A_{0} P e_{1} \approx r_{1}$, where $r_{1} \equiv R r_{0}$ is the coarse-grained residual. So this is the equation we should aim to solve on the coarse grid, i.e. we should take $A_{1}=R A_{0} P$. (Note that this derivation does not need the condition $R P=I$ 19, 22, 23, which in fact is meaningless.) This derivation holds whether or not $A_{0}$ is symmetric and/or positive-definite, and whether or not $R=P^{*}$.

A slight variant of this argument, using the $=$ sign instead of $\approx$, goes as follows: In the coarsegrid-correction phase of the multigrid algorithm, we replace $x_{0}$ by $x_{0}+P x_{1}$ for some $x_{1} \in V_{1}$. How should we choose $x_{1}$ ? Ideally we would like the new vector $x_{0}+P x_{1}$ to have a zero residual, i.e. $A_{0}\left(x_{0}+P x_{1}\right)-b=0$; but this is obviously too much to hope for in general, since $\operatorname{dim} V_{1}<$ $\operatorname{dim} V_{0}$. The best we can hope for is to annihilate the smooth part of the residual, i.e. to have $R\left[A_{0}\left(x_{0}+P x_{1}\right)-b\right]=0$. (As before, we expect that the non-smooth part of the residual has already been made small by the fine-grid relaxation.) We should therefore aim to choose $x_{1}$ so that $R A_{0} P x_{1}=-r_{1}$.

13 If the fine-grid operator $A_{0}$ is symmetric (resp. symmetric and positive-semidefinite) and we make the variational choices $R=P^{*}$ and $A_{1}=R A_{0} P$, then the coarse-grid operator $A_{1}$ is likewise symmetric (resp. symmetric and positive-semidefinite) - a useful property.

14 If the fine-grid operator $A_{0}$ is symmetric and positive-definite, then we can give a strong argument [39, pp. 56-57] [40, p. 2043] for the variational choices $R=P^{*}$ and $A_{1}=R A_{0} P$. Note first that solving $A_{0} x=b$ is equivalent to minimizing the energy functional $H_{0}(x)=\frac{1}{2}\left\langle x, A_{0} x\right\rangle-$ $\langle b, x\rangle$. Now, in the coarse-grid-correction phase of the multigrid algorithm, we replace the current approximate solution $x_{0}$ by a hopefully better approximate solution $x_{0}+P x_{1}$, where $x_{1} \in V_{1}$. A sensible goal is to attempt to choose $x_{1}$ so as to minimize $H_{0}$; this means that we attempt to minimize $H_{1}\left(x_{1}\right) \equiv H_{0}\left(x_{0}+P x_{1}\right)=\frac{1}{2}\left\langle x_{1}, P^{*} A_{0} P x_{1}\right\rangle-\left\langle P^{*}\left(b-A_{0} x_{0}\right), x_{1}\right\rangle+$ const. This is precisely what the multigrid algorithm with $R=P^{*}$ and $A_{1}=P^{*} A_{0} P$ does. (The only loophole in this logic is that one might entertain the possibility of "coarse-grid overcorrection" 41, 12, 18, 24, i.e. of choosing temporarily a sub-optimal approximate solution $x_{1}$ in the hope that this will later bring benefits.) 


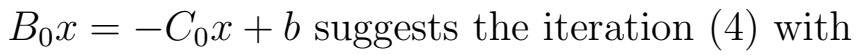

$$
\begin{aligned}
& S_{0}=-B_{0}^{-1} C_{0}=I-B_{0}^{-1} A_{0} \\
& T_{0}=B_{0}^{-1}
\end{aligned}
$$

[Conversely, any convergent first-order stationary linear iteration (团) arises from a splitting $B_{0}=T_{0}^{-1}=A_{0}\left(I-S_{0}\right)^{-1}$.] A "good" choice of $B_{0}$ (for a single-grid algorithm) is one that is "as close to $A_{0}$ as possible", subject to the condition that it be "easy to invert": this makes $S_{0}$ as small as possible.

Let us now examine the oft-made statement that "the slow modes of the (damped) Jacobi iteration correspond to the low eigenvalues of $A_{0}$ ". This statement is in fact meaningless, because $A_{0}$ does not map a space into itself, and thus does not have eigenvalues. But it is easy to see what the correct statement is: the slow modes of (田) correspond to the eigenvalues near 1 of $S_{0}$, hence to the low eigenvalues of $B_{0}^{-1} A_{0}$. Here $B_{0}^{-1} A_{0}$ maps $V_{0}$ into itself, and so does have eigenvalues.

But we can also understand how the meaningless statement could have arisen. The (damped) Jacobi iteration is defined by letting $B_{0}$ be (a multiple of) the diagonal part of $A_{0}$ with respect to some particular basis. Now, for certain operators $A_{0}$ in certain bases - for example, the Laplace operator $-\beta \Delta_{U}+m^{2}$ in a site-wise basis - it happens that the diagonal part of $A_{0}$ is given by a multiple of the identity matrix, so it is easy to think (incorrectly) that we are discussing the eigenvalues of $A_{0}$ rather than those of $B_{0}^{-1} A_{0}$. I emphasize that the performance of the Jacobi algorithm is a property not only of $A_{0}$ but also of the choice of basis: for example, for the same Laplace operator $A_{0}=-\beta \Delta_{U}+m^{2}$ with $U \equiv I$, if we use a Fourier basis we have $B_{0}=A_{0}$, in which case the relaxation process has no slow modes. On the other hand, for more general operators $A_{0}$ - such as Laplace operators with a site-dependent mass term - the diagonal part of $A_{0}$ is not a multiple of the identity matrix, even in the site-wise basis; so in such a case the conventional statement is not merely misleading but is in fact incorrect. In all cases, the relevant quantities are the eigenvalues and eigenvectors of $B_{0}^{-1} A_{0}$.

Returning to the multigrid algorithm, we can summarize the situation as follows:

i) The fine-grid problem is specified by an operator $A_{0}: V_{0} \rightarrow V_{0}^{*}$, which is usually symmetric and positive-definite (as will be assumed henceforth). No other structure is present in the original problem.

ii) Choosing a fine-grid relaxation method amounts to choosing a second operator $B_{0}: V_{0} \rightarrow V_{0}^{*}$. This operator is often (but not always) symmetric and positivedefinite. ${ }^{\text {Tu }}$

15 The (damped) Jacobi choice of $B_{0}$ is symmetric and positive-definite. The Gauss-Seidel or SOR choice of $B_{0}$ is usually not symmetric, but it may be made symmetric (as well as positivesemidefinite) by explicit symmetrization, e.g. a forward sweep followed by a reverse sweep. Note also that $B_{0}$ is symmetric if and only if $S_{0} \equiv I-B_{0}^{-1} A_{0}$ is $A_{0}$-symmetric (this means that $A_{0} S_{0}=S_{0}^{*} A_{0}$ ). 
We must then choose the remaining elements of the multigrid algorithm: $R, P$ and $A_{1}$. Obviously these choices can and should depend on $A_{0}$ and $B_{0}$; but they should not depend on any additional structures unless we have a good physical or mathematical reason for introducing such structures into the problem.

In footnotes 1214 above, I have given strong arguments for the variational choices $R=P^{*}$ and $A_{1}=P^{*} A_{0} P$. Henceforth these choices will be assumed. The only remaining choice is therefore that of the interpolation operator $P$. (This is, of course, the heart of the problem is disordered systems.)

The goal in the choice of $P$ is obviously to maximize the speed of convergence of the algorithm, measured in CPU-time units. Now, the asymptotic rate of convergence of any first-order stationary linear iteration is $-\log \rho(M)$, where $\rho(M)$ is the spectral radius multigrid algorithm is rather complicated - it depends on the number of levels, on the choice of $\mathrm{V}$-cycle or $\mathrm{W}$-cycle, etc. - so it is convenient to consider instead the iteration matrix of the idealized two-grid algorithm, i.e. the two-grid algorithm in which one assumes that the coarse-grid equation $A_{1} x_{1}=b_{1}$ is solved exactly. This is a useful warm-up problem prior to studying the complete multigrid algorithm, and there are both heuristic and rigorous connections between the two algorithms. $\square^{17}$ Let us therefore concentrate henceforth on the iteration matrix of the idealized two-grid algorithm, for simplicity with only pre-smoothing:

$$
M_{0}=\left(I-P A_{1}^{-1} R A_{0}\right) S_{0} .
$$

It is worth noting that the operator $P A_{1}^{-1} R A_{0}: V_{0} \rightarrow V_{0}$ is the $A_{0}$-orthogonal projection onto the range of $P$.

The condition for minimizing the spectral radius of $M_{0}$ was given some years ago by Greenbaum [45]: If $\operatorname{dim} V_{1}=n_{1}$, then $\rho\left(M_{0}\right)$ is minimized when Ran $P$ consists of

16 The spectral radius of an operator $M: V \rightarrow V$ is the largest absolute value of an eigenvalue.

17 Obviously the good performance of the idealized two-grid algorithm is a necessary condition for the good performance of the multigrid algorithm. On the other hand, it is also a sufficient condition, at least for the $\mathrm{W}$-cycle: if the energy norm of the idealized-two-grid iteration matrix is $\leq(\sqrt{2} / 2)-\epsilon$ uniformly in the lattice size, then the energy norm of the multigrid iteration matrix is $\leq 1-\delta$ uniformly in the lattice size [43, 44]. (The energy norm of an operator $M: V_{0} \rightarrow V_{0}$ is $\|M\|=\sup _{x \in V_{0}}\left(\left\langle M x, A_{0} M x\right\rangle /\left\langle x, A_{0} x\right\rangle\right)^{1 / 2}$.)

${ }^{18}$ First proof (computational): Clearly Ran $P A_{1}^{-1} R A_{0} \subset \operatorname{Ran} P$. To show that $P A_{1}^{-1} R A_{0}$ acts as the identity on Ran $P$, let us apply it to a vector $x_{0}=P x_{1}$ where $x_{1} \in V_{1}$ : we get $P A_{1}^{-1} R A_{0} P x_{1}=$ $P x_{1}$ since $R A_{0} P=A_{1}$. Finally, we note that $P A_{1}^{-1} R A_{0}$ is $A_{0}$-symmetric, i.e. $A_{0}\left(P A_{1}^{-1} R A_{0}\right)=$ $\left(P A_{1}^{-1} R A_{0}\right)^{*} A_{0}$; this is an immediate consequence of $A_{0}^{*}=A_{0}, A_{1}^{*}=A_{1}$ and $R=P^{*}$.

Second proof (conceptual): By construction, the coarse-grid-correction phase of the idealized twogrid algorithm with the variational choices $R=P^{*}$ and $A_{1}=P^{*} A_{0} P$ replaces the vector $x_{0}$ by that vector $x_{0}+P x_{1}\left(x_{1} \in V_{1}\right)$ which minimizes the energy $\frac{1}{2}\left\langle\left(x_{0}+P x_{1}\right), A_{0}\left(x_{0}+P x_{1}\right)\right\rangle$. (Here we can assume without loss of generality a right-hand side $b=0$.) This means that the operator $I-P A_{1}^{-1} R A_{0}$ is the $A_{0}$-orthogonal projection onto the $A_{0}$-orthogonal complement of Ran $P$. 
the $n_{1}$ eigenvectors of $B_{0}^{-1} A_{0}$ with the smallest eigenvalues. of the interpolation is constrained here: we can compose $P$ with an arbitrary bijective map in $V_{1}$, and as long as we use the variational choices $R=P^{*}$ and $A_{1}=P^{*} A_{0} P$, this merely amounts to a redefinition of the coarse-grid fields, which has no effect on the sequence of approximants $x_{0}$ that the algorithm computes.

To be sure, Greenbaum's condition is "impractical", for two reasons: Firstly, it is usually unfeasible to compute the eigenvectors of $B_{0}^{-1} A_{0}$ (unless $A_{0}$ and $B_{0}$ are constant-coefficient difference operators), as this would take a CPU time of order (volume) $)^{3}$. Secondly, even if we could compute the eigenvectors of $B_{0}^{-1} A_{0}$, we would not want to use them as the columns of the interpolation matrix, because the matrices $P, R$ and $A_{1}$ would then be dense rather than sparse, and each iteration of the resulting multigrid algorithm would take a CPU time of order (volume) ${ }^{2}$. Nevertheless, Greenbaum's condition is important because it indicates the ideal towards which we are striving, even though in practice this ideal will have to be traded off against considerations of computational complexity.

It is in this context that we can consider the Hamburg group's recent proposal [22, 23], which goes as follows: The "best" idealized two-grid algorithm is the one with the smallest spectral radius of $M_{0}$. But spectral radii are difficult to compute, so let us study instead some norm of $M_{0}$; this changes the problem, but hopefully not by too much. Now, norms are often difficult to compute, too; but one norm which is easy to compute is the Frobenius (Hilbert-Schmidt) norm $\left\|M_{0}\right\|_{2}^{2}=\operatorname{tr} M_{0} M_{0}^{*}$. The first trouble - as the reader should by now be able to guess - is that this expression is meaningless: the range of $M_{0}^{*}$ is in $V_{0}^{*}$, while the domain of $M_{0}$ is $V_{0}$. However, we can fix it by defining instead

$$
\begin{aligned}
\left\|M_{0}\right\|_{2}^{2} & =\operatorname{tr} B_{0} M_{0} B_{0}^{-1} M_{0}^{*} \\
(= & \left.\operatorname{tr} M_{0} B_{0}^{-1} M_{0}^{*} B_{0}=\operatorname{tr} B_{0}^{-1} M_{0}^{*} B_{0} M_{0}=\operatorname{tr} M_{0}^{*} B_{0} M_{0} B_{0}^{-1}\right) .
\end{aligned}
$$

This expression is meaningful without regard to any choice of basis, and it agrees with the preceding expression in any basis in which $B_{0}$ is represented by a multiple of the identity matrix.²

But the trouble is really more fundamental: Why are we trying to minimize (8), anyway? Minimizing (8) with respect to $P$ (with $R$ and $A_{1}$ fixed to the variational choices) is really no easier than minimizing the spectral radius (as far as I can tell);

${ }^{19}$ Actually, Greenbaum's condition 45 is slightly different, as she seeks to minimize the energy norm of $M_{0}$ (see footnote 17 for its definition) rather than the spectral radius. The two criteria are equivalent whenever $B_{0}$ is symmetric (i.e. $S_{0}$ is $A_{0}$-symmetric).

${ }^{20}$ A similar use of $B_{0}$ - or more precisely, of the diagonal part of $A_{0}$ - can be found in the norms defined by Ruge and Stüben [46, p. 78].

${ }^{21}$ Such a basis can always be achieved by a $G L(N, \mathbb{C})$ gauge transformation $A_{x y} \rightarrow U_{x} A_{x y} U_{y}^{*}$. If the diagonal elements $A_{x x}$ are multiples of the identity in color space, it can be achieved simply by a space-dependent rescaling $A_{x y} \rightarrow \lambda_{x} A_{x y} \lambda_{y}^{*}$. 
and even if it were, we wouldn't want to use the answer anyway, because it isn't sparse. Basically, the Hamburg condition - even if modified to map to the right spaces - suffers from the same defects as Greenbaum's original condition, without having its strongest virtues.22

The real goal, I believe, is to minimize $\rho\left(M_{0}\right)$ [or $\left\|M_{0}\right\|_{2}$ or something like it], subject to some constraint on the computational complexity of the resulting algorithm. There seem to be two main approaches:

1) Geometric multigrid. Fix the sparsity pattern of $P$ (and thus of $A_{1}$ ), and minimize $\rho\left(M_{0}\right)$ subject to this constraint. This amounts to fixing the spatial geometry of the coarse grids and the interpolation.

2) Algebraic multigrid (AMG) [46]. Constrain roughly the number of nonzero entries in $P$ and $A_{1}$, but allow the algorithm to select the sparsity pattern of $P$ (and thus of $A_{1}$ ) based on some analysis of the matrices $A_{0}$ and $B_{0}$.

The AMG approach is potentially more powerful, but also more complicated. In cases of very strong disorder, such as the random-resistor problem [1, 21, AMG is probably essential; but for gauge fields that are moderately smooth, the geometric approach may possibly work, and it makes sense to try it first. The problem of minimizing $\rho\left(M_{0}\right)$ [or some proxy for it] subject to a constrained sparsity pattern for $P$ needs to be examined from first principles.

Some readers may find the distinction between $V$ and $V^{*}$ to be "pedantic" or "academic". I can say only that I have found this distinction to be an important clarifying principle - analogous to the distinction between meters and kilograms, or between contravariant and covariant tensors in general relativity 23 - which helps in sorting out the kernel of meaning amid a mass of formulae.

${ }^{22}$ In 22, 23 the Hamburg group actually does something different from what I have just described: Firstly, instead of $\left\|M_{0}\right\|_{2}$ they consider $\left\|A_{0}^{-1} M_{0} S_{0}^{-1}\right\|_{2}$. Secondly, they fix $R$ (without justifying their choice by any optimality condition), and then minimize (8) with respect to $P$, imposing $R P=I$ and the Galerkin condition $A_{1}=R A_{0} P$ but obviously not the condition $R=P^{*}$. I do not understand the logic behind either of these elements of their approach.

${ }^{23}$ For example, the 4-velocity of a fluid is naturally a vector field, while the electromagnetic vector potential is naturally a covector field (=1-form). In the presence of a metric tensor one can of course convert freely back and forth between vectors and covectors ("raising and lowering indices"); but the question is whether this operation is physically natural in the given context (that is, whether the physics of the problem at hand has anything to do with a spacetime metric). By insisting that raising-lowering operations be indicated explicitly, one facilitates such an inquiry. More generally, the goal is to highlight the physically relevant structures, and to eliminate the irrelevant ones. Does the problem at hand really need all the information in a metric? Can it perhaps be formulated on a differentiable manifold with no additional structure? Or on a manifold with only a conformal structure? Or on a manifold with only an affine connection? And so forth.

${ }^{24}$ In the multigrid literature, the distinction between $V$ and $V^{*}$ can be found in 40, Sections II.B and II.C] and [47, Section 8]. Possibly there are also other references of which I am unaware. 
Other readers may object to my advocacy of a basis-independent approach on the grounds that "the computer must of necessity work in a particular basis". This is of course true, just as it is true that the article you are now reading must of necessity be written in a particular natural language (in this case English). Nevertheless, the content of this article should remain the same if, for example, it is translated to SerboCroatian; likewise, the content of the multigrid computation should remain the same if it is translated to another basis. The basis has no more physical relevance than does the choice of $(x, y, z)$ axes in mechanics. In reality, the basis has only two relevant effects: on roundoff error, and on computational complexity. The former effect is quite easily handled by making rescalings (or $G L(N, \mathbb{C})$ gauge transformations) to prevent the matrix elements from getting too big or too small; this is a mere computational detail, which does not alter the underlying algorithm. The computational complexity is, on the other hand, a serious issue: as mentioned above, it must be handled by imposing some constraints on the sparsity pattern of $P$.

Fermions. The reader is probably wondering how fermions fit into the foregoing framework. The answer is that I am not completely sure, but here is my best attempt:

The underlying structure of a fermionic model seems to be a pair of vector spaces $V, \bar{V}$ equipped with a distinguished bijective map $E: V \rightarrow \bar{V}$ (here $E$ maps $\psi_{i}$ onto its corresponding field $\bar{\psi}_{i}$ ). ${ }^{2}$ The fermionic operator $A=\not D+m$ then maps $V \rightarrow \bar{V}^{*}$ (think of the bilinear form $\bar{\psi}_{i} A_{i j} \psi_{j}$ ).

There are two main approaches to multigrid for fermions:

1) Work directly with the fermionic operator $A$ [11, 14, 26, 27, 28, 29, 30, 31, 32, 33, 34, 35.

2) Work with the "bosonic" operator $A^{*} A$ or $A A^{*}[6$, 7, 8, 9, 10, 19, 20, 21, 25].

My personal preference is for the second approach: it leads us back to the familiar terrain of a symmetric positive-definite operator, for which we can confidently apply Gauss-Seidel smoothing, the variational choices $R=P^{*}$ and $A_{1}=P^{*} A_{0} P$, and so forth; the sole question is the usual one, namely the choice of the interpolation $P$.

The only trouble with the operators $A^{*} A$ and $A A^{*}$ is that, according to my framework, they are meaningless! If $A$ maps $V \rightarrow \bar{V}^{*}$, then $A^{*}$ maps $\bar{V} \rightarrow V^{*}$, so the combinations $A^{*} A$ and $A A^{*}$ are meaningless. Worse yet, there is no way of making a meaningful "squared" map $V \rightarrow V^{*}$ or $\bar{V} \rightarrow \bar{V}^{*}$ (remember that we want it to be positive-semidefinite, so it has to map a space into its dual) out of the ingredients $A, A^{*}, E, E^{*}, E^{-1}$ and $\left(E^{-1}\right)^{*} !$ Rather, it seems that one may consider, with equal justice, any of the operators $A^{*} K A$ or $A L A^{*}$, where $K: \bar{V}^{*} \rightarrow \bar{V}$ and $L: V^{*} \rightarrow V$ are symmetric and positive-definite but otherwise arbitrary.

\footnotetext{
${ }^{25}$ Equivalently, one can consider the vector space $V \oplus \bar{V}$ equipped with a distinguished involution $J: V \oplus \bar{V} \rightarrow V \oplus \bar{V}$, where $J=\left(\begin{array}{cc}0 & E^{-1} \\ E & 0\end{array}\right)$.
} 
Luckily, this formalism need be applied only to the original (fine-grid) fermion operator, which is a Wilson or staggered fermion operator in a unitary gauge field and with a space-independent color-independent mass term. (The coarse-grid operators, which may well involve "dielectric" gauge fields, are all bosonic.) Therefore, it may be possible to justify the standard choice of $K$ and $L$ - namely, the operator specified by the identity matrix in the site-wise basis - as being in some precise sense "natural". I leave this as a open problem.

Some concluding remarks. The foregoing comments should not be interpreted as a dismissal of the existing literature on multigrid methods for propagators. In fact, I believe that the two major existing approaches - parallel-transported multigrid [26, 27, 28, 29, 30, 31, 32, 33, 34, 35] and ground-state-projection multigrid [4, 5, 6, 8, 9, 11, 12, 13, 14, 15, 17, 18, 19, 20, 21, 24, 25 — both contain valuable physical insights that are likely to play a role in successful future algorithms. However, I believe that these methods must be rethought and reworked to bring them into conformity with the three basic conceptual principles expounded here.

Some readers will undoubtedly feel that these conceptual principles - especially the distinction between $V$ and $V^{*}$ - are mere "mathematical nit-picking", and are irrelevant to the practical task of designing an efficient algorithm. I take the opposite point of view: it seems to me that at the current stage of development, we have already too many proposals for multigrid algorithms and their ingredients, not too few; what we need now is to improve our understanding of what we are doing, so as to lay a solid foundation for future success in practice. Only time will tell.

I wish to thank Radi Ben-Av, Rich Brower, Robert Edwards, Jonathan Goodman, Thomas Kalkreuter, Gerhard Mack, Sorin Solomon and Marcus Speh for numerous discussions over the past few years. This research was supported in part by National Science Foundation grant DMS-9200719.

\section{References}

[1] R.G. Edwards, J. Goodman and A.D. Sokal, Phys. Rev. Lett. 61, 1333 (1988).

[2] R.G. Edwards, J. Goodman and A.D. Sokal, Nucl. Phys. B (Proc. Suppl.) 9, 521 (1989).

[3] R.G. Edwards, "Numerical Simulations in Lattice Gauge Theories and Statistical Mechanics", Ph.D. thesis, New York University (April 1989).

[4] A. Hulsebos, J. Smit and J.C. Vink, Nucl. Phys. B (Proc. Suppl.) 9, 512 (1989). 
[5] A. Hulsebos, J. Smit and J.C. Vink, Nucl. Phys. B331, 531 (1990).

[6] A. Hulsebos, J. Smit and J.C. Vink, Nucl. Phys. B (Proc. Suppl.) 20, 94 (1991).

[7] J.C. Vink, Phys. Lett. B272, 81 (1991).

[8] A. Hulsebos, J. Smit and J.C. Vink, Nucl. Phys. B368, 379 (1992).

[9] A. Hulsebos, J. Smit and J.C. Vink, Int. J. Mod. Phys. C3, 161 (1992).

[10] J.C. Vink, Nucl. Phys. B (Proc. Suppl.) 26, 607 (1992).

[11] R.C. Brower, C. Rebbi and E. Vicari, Phys. Rev. D43, 1965 (1991).

[12] R.C. Brower, K.J.M. Moriarty, C. Rebbi and E. Vicari, Phys. Rev. D43, 1974 (1991).

[13] R.C. Brower, C. Rebbi and E. Vicari, Phys. Rev. Lett. 66, 1263 (1991).

[14] R.C. Brower, R.G. Edwards, C. Rebbi and E. Vicari, Nucl. Phys. B366, 689 (1991).

[15] R. Brower, K. Moriarty, C. Rebbi, E. Vicari, Nucl. Phys. B (Proc. Suppl.) 20, 89 (1991).

[16] G. Mack, in Nonperturbative Quantum Field Theory, ed. G. 't Hooft et al. (Plenum Press, New York, 1988).

[17] T. Kalkreuter, Nucl. Phys. B376, 637 (1992).

[18] T. Kalkreuter, Phys. Lett. B276, 485 (1992).

[19] T. Kalkreuter, G. Mack and M. Speh, Int. J. Mod. Phys. C3, 121 (1992).

[20] T. Kalkreuter, Int. J. Mod. Phys. C3, 1323 (1992).

[21] T. Kalkreuter, Nucl. Phys. B (Proc. Suppl.) 30, 257 (1993).

[22] M. Bäker, T. Kalkreuter, G. Mack and M. Speh, Int. J. Mod. Phys. C4, 239 (1993).

[23] M. Bäker, G. Mack and M. Speh, Nucl. Phys. B (Proc. Suppl.) 30, 269 (1993).

[24] T. Kalkreuter, Multigrid methods for the computation of propagators in gauge fields, DESY preprint 92-158 (November 1992), hep-lat@ftp.scri.fsu.edu \#9212021. A shortened version of this paper has been submitted to Int. J. Mod. Phys. C. 
[25] T. Kalkreuter, Idealized multigrid algorithm for staggered fermions, DESY preprint 93-046 (April 1993), hep-lat@ftp.scri.fsu.edu \#9304004.

[26] R. Ben-Av, A. Brandt and S. Solomon, Nucl. Phys. B329, 193 (1990).

[27] R. Ben-Av, A. Brandt, M. Harmatz, E. Katznelson, P.G. Lauwers, S. Solomon and K. Wolowesky, Phys. Lett. B253, 185 (1991).

[28] M. Harmatz, P.G. Lauwers, R. Ben-Av, A. Brandt, E. Katznelson, S. Solomon and K. Wolowesky, Nucl. Phys. B (Proc. Suppl.) 20, 102 (1991).

[29] R. Ben-Av, "Acceleration Methods in MC Simulations", Ph.D. thesis, Weizmann Institute of Science (October 1991).

[30] P.G. Lauwers, R. Ben-Av and S. Solomon, Nucl. Phys. B374, 249 (1992).

[31] P.G. Lauwers and S. Solomon, Int. J. Mod. Phys. C3, 149 (1992).

[32] M. Harmatz, P.G. Lauwers, S. Solomon and T. Wittlich, Nucl. Phys. B (Proc. Suppl.) 30, 192 (1993).

[33] P.G. Lauwers and T. Wittlich, Nucl. Phys. B (Proc. Suppl.) 30, 261 (1993).

[34] P.G. Lauwers and T. Wittlich, Int. J. Mod. Phys. C4, 609 (1993).

[35] R. Ben-Av, M. Harmatz, P.G. Lauwers and S. Solomon, Parallel-transported multigrid for inverting the Dirac-operator: Variants of the method and their efficiency, Arbeitspapiere der GMD 674 (August 1992), to appear in Nucl. Phys. B.

[36] G. Mack, Nucl. Phys. B235, 197 (1984).

[37] S.F. McCormick and J. Ruge, Math. Comput. 41, 43 (1983).

[38] W.L. Briggs, A Multigrid Tutorial (SIAM, Philadelphia, 1987).

[39] A. Brandt, Multigrid Techniques: 1984 Guide with Applications to Fluid Dynamics, GMD-Studien No. 85 (Gesellschaft für Mathematik und Datenverarbeitung mbH Bonn, May 1984).

[40] J. Goodman and A.D. Sokal, Phys. Rev. D40, 2035 (1989).

[41] T. Meis and H.-W. Branca, Z. Angew. Math. Mech. 62, T263 (1982).

[42] J. Goodman and L. Reyna, private communication.

[43] S.F. McCormick and J.W. Ruge, SIAM J. Numer. Anal. 19, 924 (1982). 
[44] J.-F. Maitre and F. Musy, SIAM J. Numer. Anal. 21, 657 (1984).

[45] A. Greenbaum, SIAM J. Numer. Anal. 21, 473 (1984).

[46] J.W. Ruge and K. Stüben, in Multigrid Methods, ed. S.F. McCormick (SIAM, Philadelphia, 1987), pp. 73-130.

[47] J.H. Bramble, J.E. Pasciak, J. Wang and J. Xu, Math. Comp. 57, 23 (1991). 